\title{
Marking and bridging versus conditioned reinforcement
}

\author{
BEN A. WILLIAMS \\ University of California, San Diego, La Jolla, California
}

\begin{abstract}
Rats were trained on a series of reversals of a two-choice conditional discrimination. Choice responses were followed by different delays of reinforcement, which were either unsignaled or filled with either a brief or a long tone. In some conditions, the tone occurred following both correct and incorrect choices; in other conditions, the tone occurred only after correct choices. Presentation of the tone following only correct choices greatly facilitated the acquisition of the discrimination, and there was little effect of the tone's duration. Presentation of the tone following all choices did not improve discrimination acquisition relative to the no-signal condition. The results demonstrate facilitatory effects of a signal during a delay-of-reinforcement interval that are caused by the conditioned-reinforcement properties of the signal and cannot be explained by the alternative mechanisms of marking or bridging.
\end{abstract}

Stimuli presented during a delay-of-reinforcement interval are known to facilitate learning, in contrast to when the delay interval is unsignaled (Spence, 1947). This facilitatory effect has been traditionally interpreted in the context of conditioned reinforcement. The stimulus is itself temporally contiguous with reinforcer delivery, which results in its acquiring reinforcement properties of its own because of the Pavlovian contingency. Then the stimulus reinforces the response on which its presentation is immediately contingent.

Although there are numerous demonstrations of the validity of the concept of conditioned reinforcement (e.g., Royalty, Williams, \& Fantino, 1987; Williams \& Dunn, 1991a, 1991b), several alternative mechanisms have been proposed to answer the question of why a stimulus intervening in a delay-of-reinforcement interval exerts its effect (see Kaplan \& Hearst, 1982, for an excellent discussion). One alternative that has received considerable empirical support is the concept of marking (Lieberman, Davidson, \& Thomas, 1985; Lieberman, McIntosh, \& Thomas, 1979). The critical difference between procedures that have demonstrated marking and those that have studied conditioned reinforcement is that in marking procedures, the stimulus is typically presented only at the start of the delay interval and is contingent on both correct and incorrect responses in a two-choice discrimination. Thus, any conditioned-reinforcement effects of the stimulus should be equated for the two responses, so that no differential response-strengthening caused by conditioned reinforcement should occur. Nevertheless, marking procedures have been shown to facilitate substantially

This research was sponsored by NIMH Grant MH 42797 and an NSF grant to the University of California, San Diego. Reprint requests should be sent to the author, Department of Psychology, University of California, San Diego, La Jolla, CA 92093-0109. the rate of learning, in contrast to when no signal is presented during the delay. The interpretation that is given to this facilitation is that the brief stimulus perceptually isolates the choice response, thus making it more salient in memory at the time the response consequence is eventually delivered at the end of the delay interval.

The presentation of the signal that is contingent on both correct and incorrect choices in a marking procedure is different from the standard-conditioned-reinforcement procedure in which the signal is presented only after correct choices. Given that conditioned reinforcement is not a sufficient explanation of marking effects, the issue raised is whether marking effects themselves explain the presumed effects of conditioned reinforcement. That is, given a procedure in which the signal occurs only after a correct response, perhaps the facilitation produced by the signal is not due to the signal's value per se but instead occurs because the signal perceptually isolates the operant response. Such an interpretation has been given to a variety of signal effects on operant behavior (e.g., Reed, 1989; Reed \& Hall, 1989). Thus, to the extent that marking effects are pervasive, the necessity of the concept of conditioned reinforcement diminishes.

A second alternative to the concept of conditioned reinforcement has been labeled "catalysis" by Rescorla (1982). The basic notion is that a stimulus intervening between a CS and a US may facilitate the CS-US association by serving as a bridge over the temporal gap. This facilitation need not depend on the value of the intervening stimulus but may occur simply because "events which are bridged in time by a third event appear to go together" (Rescorla, 1982, p. 140). This "bridging"' hypothesis was supported by Rescorla with several experiments, the design of the most critical of which (Experiment 4) is shown in Table 1. Two different target stimuli were followed after a temporal gap by the reinforcer on $50 \%$ of the trials. The stimuli were each followed immediately by the same 
Table 1

Design of Experiment 4 of Rescorla (1982)

\begin{tabular}{ccc}
\hline $\begin{array}{c}\text { Target } \\
\text { Stimulus }\end{array}$ & Gap & Reinforcer \\
\hline A & $\mathrm{X}$ & US \\
A & - & - \\
B & $\bar{x}$ & US \\
B & - \\
\hline
\end{tabular}

signal on $50 \%$ of the trials, so that any conditionedreinforcement effects of the signal should have been equated for both of the target stimuli. The critical difference between the two targets is that both the signal and the reinforcer occurred on the same trials for Stimulus A and on different trials for Stimulus B. The rationale was that Stimulus A should be more likely to be perceptually linked to the reinforcer because the signal bridged the temporal gap. In keeping with this hypothesis, greater conditioning occurred to Stimulus A.

As with the concept of marking, the critical feature of the experimental design in Table 1 that distinguishes the concept of bridging from that of conditioned reinforcement is that the intervening signal follows the two target elements on an equal number of trials. Thus, any difference in the associative strength of the two CSs presumably cannot be explained by conditioned reinforcement. Also, as with the marking concept, the demonstration of the occurrence of bridging with such procedures raises the possibility that bridging is also involved in procedures in which the signal follows only one of the target elements, and which previously have been interpreted as evidence for conditioned reinforcement. Once again, therefore, such demonstrations diminish the need for the concept of conditioned reinforcement.

Although both the concepts of marking and bridging have impressive empirical support, the power of their effects has never been directly compared with conditioned reinforcement. That is, the facilitation seen in the marking and bridging procedures, in which the intervening stimulus occurs after both target elements, has never been directly compared to that which occurs when the signal follows only one of the target elements. Without such a comparison, it is impossible to determine whether the effects of marking and bridging are pervasive, and thus can effectively displace the concept of conditioned reinforcement, or whether they are second-order effects that are theoretically provocative but actually account for little of the variance in delay-of-reinforcement procedures. The present study was conducted to provide such a direct comparison.

Rats were presented with a series of reversals of a simultaneous conditional discrimination. On some sessions, a light signaled that responses to the left lever produced food and responses to the right lever no food, whereas a noise signaled that the right lever was correct and the left lever was incorrect. After this problem was learned to a criterion, the response contingencies that were correlated with the two conditional stimuli were reversed and the problem was again learned to criterion. Different delay-of- reinforcement contingencies were then presented over successive blocks of reversals. Such a serial reversal procedure provides a within-subjects method of studying acquisition effects that avoids the problems of intersubject variability that are often seen in between-subjects studies of acquisition (for representative examples of previous work with this procedure, see Williams, 1971, 1981).

The different conditions in the experiment were defined by the stimulus conditions that occurred during the delayof-reinforcement interval. For the no-signal condition, the choice responses were followed by the termination of the conditional stimulus and the removal of the response levers, and then the presentation of the food reinforcer at the end of the delay interval if the preceding choice had been correct. For the standard-conditioned-reinforcement condition, incorrect choices were followed by no signal, whereas correct choices were followed by a tone that continued throughout the delay interval until food delivery. For the briefconditioned-reinforcement condition, the contingencies were the same, except that the tone occurred only for a short burst immediately after a correct response and then was off for the remainder of the delay interval. The comparison of the standard- and brief-conditioned-reinforcement conditions was included because recent work has shown that brief conditioned reinforcers may produce responsestrengthening effects that are indistinguishable from those in which the signal extends throughout the delay interval (Schaal \& Branch, 1988). For the marking condition, the brief signal occurred after both correct and incorrect choices and then was off during the remainder of the delay-of-reinforcement interval. Finally, for the bridging condition, the signal extended throughout the delay interval after both correct and incorrect choices, terminating in food after a correct choice and in no food after an incorrect choice. Note that this version of the bridging procedure is conceptually analogous to that shown in Table 2 because both target elements were followed equally

Table 2 Order of Conditions for All Subjects

\begin{tabular}{ccl}
\hline Order & Delay* & \multicolumn{1}{c}{$\begin{array}{c}\text { Stimulus } \\
\text { Condition }\end{array}$} \\
\hline 1 & 3 & No Signal \\
2 & 3 & Cond. RF十 \\
3 & 3 & Bridging \\
4 & 3 & Marking \\
5 & 3 & Brief Cond. RF \\
6 & 3 & No Signal \\
7 & 6 & No Signal \\
8 & 6 & Cond. RF \\
9 & 6 & Bridging \\
10 & 6 & Marking \\
11 & 6 & Brief Cond. RF \\
12 & 6 & No Signal \\
13 & 12 & No Signal \\
14 & 12 & Marking \\
15 & 12 & Brief Cond. RF \\
16 & 12 & Bridging \\
17 & 12 & Cond. RF \\
18 & 12 & No Signal \\
\hline
\end{tabular}

*In seconds. †Conditioned reinforcement. 
often by the stimulus intervening during the delay. However, it is different because the targets were complex events (the conditional relation of which lever was correct given the stimulus present on a given trial) rather than simple CSs, and because the intervening stimulus occurred during all delay intervals rather than only during $50 \%$ of them. Each of these five conditions were presented for three different delay values: 3,6 , and $12 \mathrm{sec}$. At issue was how the rate of acquiring the successive reversals would vary with the stimulus conditions during the delay intervals.

\section{METHOD}

\section{Subjects}

Four Sprague-Dawley albino rats, approximately 11 months of age at the start of the study, were housed individually with a 14:10-h light:dark cycle. All of the subjects had participated in a previous study of conditioned reinforcement that used the same discrimination contingencies presented here (see Williams \& Dunn, 1991b, for details). Food deprivation was maintained by $1-\mathrm{h}$ access to $\mathrm{Pu}-$ rina Lab Chow immediately following the experimental sessions. Water was continuously available in the home cage.

\section{Apparatus}

A standard two-lever rat chamber, with clear acrylic side walls, and sheet metal ceiling, front and rear walls, and a grid floor, was housed inside a sound-attenuating larger chamber equipped with a ventilating fan. The interior dimensions of the chamber were $30.5 \mathrm{~cm}$ in width $\times 20.3 \mathrm{~cm}$ in height $\times 22.9 \mathrm{~cm}$ in length. Mounted on the front wall of the chamber, spaced $9 \mathrm{~cm}$ apart from side to side, were two retractable levers (BRS/LVE Model RRL015 ), which protruded $1.5 \mathrm{~cm}$ into the chamber when in operation and which required a minimum force of $0.3 \mathrm{~N}$ for depression. The only feedback for a leverpress was the sound of the microswitch inside the lever housing. Directly between and $6.5 \mathrm{~cm}$ below the levers was a food receptacle into which were dropped 45-mg Noyes pellets (improved formula $\mathbf{A}$ ), which served as the reinforcer. $\mathrm{Be}$ tween and $3.3 \mathrm{~cm}$ above the levers was a $28-\mathrm{V}$ pilot light with a glass translucent cover. A sonalert, which delivered an $88-\mathrm{dB}$ auditory signal, was mounted $4 \mathrm{~cm}$ above the left lever. In the center of the ceiling, a speaker was mounted through which a 77-dB white noise could be presented. Ambient noise level in the absence of the tone or the white noise was $72 \mathrm{~dB}$.

\section{Procedure}

The discrimination contingency entailed that either the left or the right lever was designated correct on a given trial, depending on whether the conditional cue was the white noise or the illumination of the pilot light. For some reversals, the noise signaled that the left lever was correct and the right lever was incorrect, whereas the light signaled the opposite contingencies. For the succeeding reversal, the contingencies for the two cues were reversed.

After a 30-sec intertrial interval (ITI), a trial began with the onset of one or the other conditional cue and the presentation of the response levers. To ensure that the subjects were adequately exposed to the conditional stimulus before their choice response, responses during the first $4 \mathrm{sec}$ had no effect; the location of the first response after the $4 \mathrm{sec}$ had elapsed then determined whether the trial was correct or incorrect. The levers were then withdrawn, the conditional cue was terminated, and the delay-of-reinforcement interval was begun. At the end of the delay interval, food was delivered following a correct choice; no food was delivered after an incorrect choice. Conditions then returned to those of the ITI, which was initiated at the time of the choice response (i.e., the delay value was included as part of the 30-sec ITI). As is shown in Table 2, the first series of conditions involved a 3-sec delay of reinforcement, the second series a 6-sec delay, and the final series a 12-sec delay.

Table 2 shows the order in which the different experimental conditions were presented. For the no-signal condition, the tone stimulus was not presented during the delay interval. For the conditionedreinforcement condition, the tone stimulus was presented throughout the delay interval following correct choices and was not presented after incorrect choices. For the brief-conditioned-reinforcement condition, when the delay value was $3.0 \mathrm{sec}$, the tone occurred during the first $0.5 \mathrm{sec}$ of the delay interval after a correct choice and did not occur after an incorrect choice. When the delay was either 6 or $12 \mathrm{sec}$, the signal duration was extended to $1.0 \mathrm{sec}$. For the marking condition, the brief signal was presented following both correct and incorrect choices, with the same durations as were used with the brief-conditioned-reinforcement condition. For the bridging condition, the signal extended throughout the delay interval after all choices, terminating in food after correct choices but no food after incorrect choices.

Each condition shown in Table 2 was presented until 4 consecutive reversals had been learned to criterion. Training on a given reversal continued until the subject had reached a criterion of 10 consecutively correct trials within a session. Training within a session was terminated either when the criterion was reached or when a total of 100 reinforcers had been delivered. When the latter occurred, training on the same contingencies was resumed the next day. The issue was how the number of trials required to attain the learning criterion varied with the stimulus conditions during the delay interval.

\section{RESULTS}

Only 3 of the 4 subjects completed the entire series of conditions shown in Table 2 . One subject died midway through the last set of conditions involving the 12-sec delay interval. Consequently, Figure 1 presents the data in two different formats. The mean data for all 4 subjects for the 3- and 6-sec delay conditions are shown in the top panel, and the mean data for the 3 subjects that completed all conditions are presented in the bottom panel. The general pattern of results is the same for both types of presentation, indicating that the pattern of results was generally consistent across subjects.

Two types of effects are evident from Figure 1. In general, the number of trials to criterion increased with the longer delay values, for all of the stimulus conditions. Secondly, there were consistent differences between conditions regardless of the delay value. With respect to the no-signal condition, both conditioned-reinforcement conditions substantially reduced the number of trials required, whereas neither the marking nor the bridging conditions, which were highly similar to each other, seemed to have much facilitative effect. To test these observations, separate two-way ANOVAs (condition $x$ delay) were conducted on the data shown in the top portion of the figure, which is based on data from all 4 subjects, and in the bottom portion, which is based only on the 3 subjects that completed all conditions. Because the possibility of order effects, the two replications of the no-signal condition (see Table 2) were treated as separate conditions for the purpose of the analyses, resulting in six, rather than five, different conditions for each delay. 

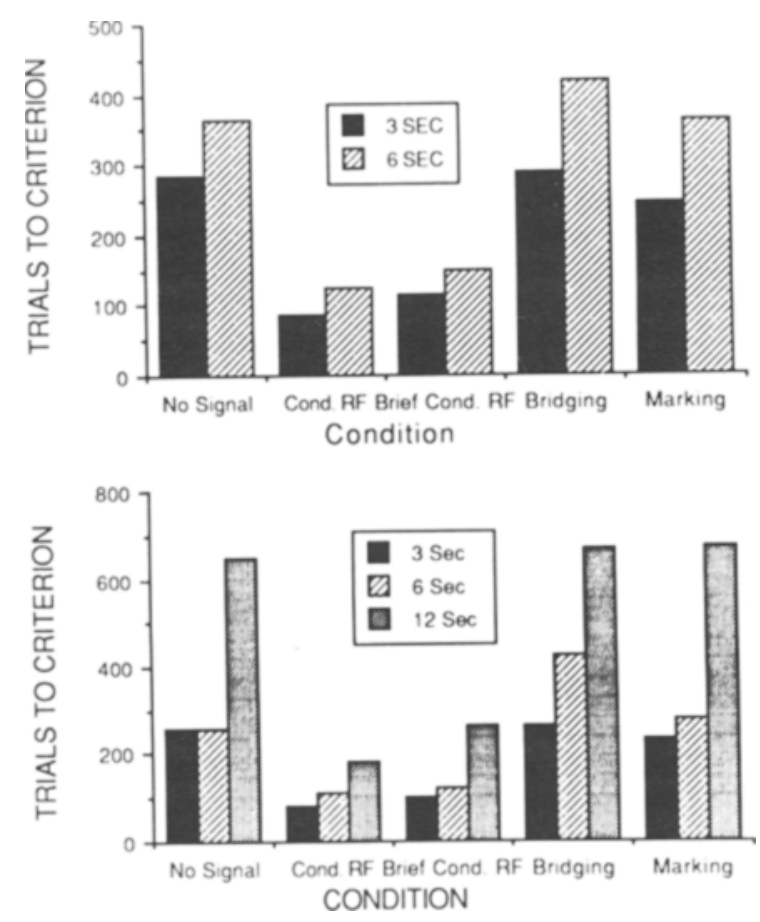

Figure 1. Mean trials to criterion required to acquire each successive reversal. The data shown are the means of four reversals per condition, except for the no-signal conditions, which were presented twice. The top portion of the graph shows the averages of all 4 subjects for the 3-and 6-sec delays. The bottom portion shows the averages of the 3 subjects that finished the entire series of conditions.

From the data in the top portion of Figure 1, it is evident that neither the effect of delay $(F<1)$ nor the interaction between delay and stimulus condition $(F<1)$ was significant. But the effect of condition was significant $[F(5,15)=8.15, p<.01]$. A comparison of the individual pairs of conditions with the use of a NewmanKeuls test (with a .05 significance level) revealed that the conditioned-reinforcement condition was significantly different from the marking, bridging, and from both no-signal conditions, as was the brief-conditioned-reinforcement condition. Neither the difference between the two conditioned-reinforcement conditions nor any of the remaining differences was significant.

The ANOVA on the data shown in the bottom portion of Figure 1 produced a similar pattern. The effect of the delay value approached but did not attain significance $[F(2,4)=4.94, .10>p>.05]$. The interaction term was not significant $[F(10,20)=1.50, p>.10]$. The effect of stimulus conditions was significant $[F(5,10)=$ $10.40, p<.01]$. Paired comparisons using the NewmanKeuls test revealed that both conditioned-reinforcement conditions were significantly different from the marking, bridging, and from both no-signal conditions, and that no other differences were significant.

The failure to achieve a significant main effect of delay may seem puzzling given the large differences in the means seen in Figure 1. Three of the 4 subjects did in fact show a monotonic relation between delay value and trials to criterion, which generally held across all of the different stimulus conditions. One subject did not; its mean trials to criterion, averaged across stimulus conditions, was smallest with the 6-sec delay and only slightly greater for the 12-sec delay than for the 3-sec delay. The most likely reason why this subject failed to show the same effect as the other three was that it improved its overall performance level with continued training, and the order of the different delay conditions, shown in Table 2, confounded the delay value with the order of presentation (i.e., the longer delays were presented later in training). Had the order of delay presentation been counterbalanced, it seems likely that an overall effect of delay would have been obtained.

To provide a closer analysis of the differences between individual stimulus conditions, the trials to criterion obtained for each delay value were averaged, and the averaged values were normalized with respect to the number obtained with the no-signal condition. The mean normalized values were $0.31,0.41,1.05$, and 0.98 for the conditioned reinforcement, brief conditioned reinforcement, bridging, and marking conditions, respectively. A comparison of the long-versus brief-conditioned-reinforcement conditions was then made with a $t$ test, with the result that the difference approached but did not obtain significance $[r(3)=2.50, .10>p>.05]$. A similar comparison was made between the bridging and the marking conditions. Here, the $t$ value did not approach significance $(t=.55)$.

\section{DISCUSSION}

The present results show that the presentation of a signal during a delay-of-reinforcement interval greatly facilitates discrimination acquisition. Compared with when the stimulus conditions during the delay were the same as the conditions of the ITI (the no-signal condition), the number of trials required for the discrimination to be learned to criterion was reduced by approximately $70 \%$. There was a similar degree of facilitation when the signal occurred only for a brief period $(0.5 \mathrm{or} 1.0 \mathrm{sec})$ at the start of the delay interval, contingent on a correct choice. There was a small difference between the brief-signal condition and the condition with the signal throughout the delay, but this difference did not attain conventional levels of significance.

The failure to find a difference as a function of signal duration is somewhat surprising, because in the briefconditioned-reinforcement condition, the signal was not temporally contiguous with food. Thus, it might be expected that its reinforcement value would be considerably less than when the signal was paired with food, and this should be reflected in the rate of learning. It should be noted that comparable effects have been obtained in freeoperant procedures in which the effects of brief and long signals have been directly compared. Schaal and Branch (1988) demonstrated that their effects were similar as long as the delay-of-reinforcement interval was not extended beyond $9 \mathrm{sec}$, at which point the longer signal became 
more effective in controlling behavior. More recently, Schaal and Branch (1990) have demonstrated that the effectiveness of the brief signal in maintaining behavior depends in part on the order of presentation of the briefversus-long signal conditions. When the long-signal conditions were presented first, followed by the brief signal, relatively little difference occurred between the two conditions, whereas presentation of the brief-signal condition first produced a much larger difference. The apparent reason for the effect of order of presentation is that signals continue to be effective conditioned reinforcers after their initial pairing with the food reinforcement, despite continued training in which they are temporally separated from the food. This observation is directly relevant to the present study because of the use of a within-subjects design. All of the subjects had experienced the tone signal temporally contiguous with the food, so that the briefsignal conditions were comparable to the conditions of Schaal and Branch (1990) in which the brief signal was presented after long-signal training. Had a betweensubjects design been used in which the brief signal had been presented without a history of temporal contiguity with the reinforcer, it seems plausible that a larger difference between the two conditioned-reinforcement conditions would have been obtained. Nevertheless, the apparent irreversibility of the effects of temporal contiguity for the reinforcement effectiveness of the brief signal is of considerable theoretical interest.

In contrast to the conditioned-reinforcement conditions, there was no facilitation of learning produced by either the bridging or the marking conditions. The large difference between the rate of learning in the conditionedreinforcement conditions as opposed to the bridging and the marking conditions clearly establishes that neither bridging nor marking can provide an adequate substitute for the concept of conditioned reinforcement as the process by which signals during delay-of-reinforcement intervals facilitate learning. However, the fact that the conditioned-reinforcement conditions produced faster learning than the bridging and the marking conditions does not necessarily prove that marking and/or bridging are ineffective, because conditioned reinforcement, bridging, and marking may all be operative in the same situation. For example, in the present situation, the signal was a potent conditioned reinforcer, so that its presentation, contingent on both correct and incorrect choices during the bridging and marking conditions, would be expected to strengthen the incorrect response and thus decrease the rate of learning. This interference effect due to conditioned reinforcement would then obscure any facilitation that bridging and marking might otherwise have had. Nevertheless, the failure to obtain any facilitation of learning by bridging or marking, with respect to the no-signal condition, suggests that neither mechanism was operative in the present situation. Even given that some interference due to conditioned reinforcement of incorrect responses occurred, there is no reason to suppose that such an ef- fect should completely overshadow the bridging/marking functions.

The failure to obtain either bridging or marking effects in the present study raises the issue of just how robust such effects actually are. Recent studies have directly challenged the necessity of the concept of bridging by further investigation of the design used by Rescorla (1982), shown in Table 1. Honey, Schachtman, and Hall (1987) replicated Rescorla's finding that more responding occurred to Target Element A than to Target Element B, but they also noted that more responding occurred to the intervening $X$ stimulus after Element $A$ as well. Similar results were obtained by Thomas, Robertson, and Cunniffe (1989), who also noted that the greater responding to $X$ following $A$ occurred even when $A$ and $X$ were presented on different response keys. They then suggested that the reason that more responding occurred to Target Element A than to Element B was that the subjects had learned that the value of $\mathrm{X}$ was conditional on the prior stimulus. Thus $\mathrm{X}$ was a potent conditioned reinforcer following A but not following B. Given such an interpretation, there is no reason to postulate the mechanism of bridging as an alternative to conditioned reinforcement. It should be noted, however, that this explanation implies a paradox. For Stimuli A and B to serve as cues for the reinforcement value of $X$, they must themselves be associated with the reinforcer in the sense that their memory must be available at the time of reinforcement presentation. In other words, Stimuli A and B must already have differential value in order for Stimulus $\mathrm{X}$ to facilitate responding to Stimulus A.

Although the present results are consistent with those of Honey et al. (1987) and Thomas et al. (1989) in challenging the necessity of invoking bridging, several important differences between the present procedure and that of Rescorla (1982) should be noted. In Rescorla's procedure, the intervening stimulus occurred on only $50 \%$ of the presentations of each CS, whereas here it occurred on all trials. Also, here the stimulus complex (presses of either the left or right levers in the presence of either the noise or the light) that was associated with the reinforcer was considerably more complex than the keylight stimuli used by Rescorla in his autoshaping procedure. Nevertheless, it is not obvious why either difference should vitiate the bridging process.

The evidence supporting the concept of marking is considerably more substantial (Lieberman et al., 1985; Lieberman et al., 1979; Lieberman \& Thomas, 1986; Thomas, Lieberman, McIntosh, \& Robertson, 1983; Thomas, Robertson, \& Lieberman, 1987). But it also should be noted that not all investigators have obtained marking effects in very similar procedures (Urcuioli \& Kasprow, 1988). Thomas and Lieberman (1990) have discussed the possible reasons for the discrepancy and have argued that marking is most likely to occur when the marking stimulus is substantially more salient than the target elements that are marked and that it is essential that 
there be close temporal contiguity between the targets and markers for marking to be effective. The failure to obtain marking in the present study possibly can be explained by the first of these factors, because the tone serving as the signal was used for a long series of conditions. Thus, even though it was a highly salient stimulus at the start of the experiment, its many sessions of presentation may have produced substantial habituation to the point that it was no longer a functionally salient event. But this interpretation is challenged by the effectiveness of the tone as a conditioned reinforcer in other conditions, an effect that remained undiminished with continued training. Indeed, the pairing of the tone with food in the conditions other than marking might be expected to maintain the tone's attentional value. Nevertheless, it is possible that relative novelty may be a critical feature of the properties of stimuli that produce the marking effect. Alternatively, the present procedure may have involved stimulus changes that overshadowed the marking effects of the tone. Each response was followed by the termination of the conditional stimuli along with the withdrawal of the response levers, and these events themselves may have produced such a substantial marking effect that any additional marking effect caused by the tone went undetected. Other factors may also have served to obscure the marking effect. For example, the repeated reversal procedure used here is quite different from the usual delayedreinforcement procedure with which marking has been studied, which typically has involved subjects learning only a single response or discrimination. Clearly, the present results fail to provide strong evidence that marking effects do not occur. The results do demonstrate, however, that powerful conditioned-reinforcement effects can be obtained in situations for which marking cannot be the explanation.

The present data provide strong evidence for the validity of conditioned reinforcement as a fundamental concept of conditioning theory. In addition to the present data, other studies using similar procedures have shown that the facilitation of learning obtained with the present procedure depends critically on the pairing of the tone signal with food, since tones unpaired with food but contingent on correct choices produced no facilitation of learning (Williams \& Dunn, 1991b). Such data strongly suggest that the power of the intervening stimulus to facilitate learning depended on the transfer of reinforcement value to that stimulus because of its temporal contiguity with food. Whatever the validity of bridging and marking as psychological concepts, the present data strongly support the old-fashioned notion of conditioned reinforcement as an essential part of any adequate theory of learning.

\section{REFERENCES}

Honey, R. C., Schachtman, T. R., \& Hall, G. (1987). Partial reinforcement in serial autoshaping: The role of attentional and associative factors. Learning \& Motivation, 18, 288-300.
KAPLAN, P. S., HEARST, E. (1982). Bridging temporal gaps between the CS and US in autoshaping: Insertion of other stimuli before, during, and after CS. Joumal of Experimental Psychology: Animal Behavior Processes, 8, 187-203.

Lieberman, D. A., Davidson, F. H., Thomas, G. V. (1985). Marking in pigeons: The role of memory in delayed reinforcement. Journal of Experimental Psychology: Animal Behavior Processes, 11. 611-624.

Lieberman, D. A., McIntosh, D. C., Thomas, G. V. (1979). Learning when reward is delayed: A marking hypothesis. Journal of Experimental Psychology: Animal Behavior Processes, 5, 224-242.

Lieberman, D. A., Thomas, G. V. (1986). Marking, memory, and superstition in the pigeon. Quarterly Joumal of Experimental Psychology, 38B, 449-459.

REED, P. (1989). Marking effects in instrumentnal performance on DRH schedules. Quarterly Joumal of Experimental Psychology, 41B, 337-353.

ReEd, P., \& HALL, G. (1989). The quasi-reinforcement effect: The influence of brief stimuli uncorrelated with reinforcement on variableratio schedules. Leaming \& Motivation, 20, 242-261.

RESCORLA, R. A. (1982). Effect of a stimulus intervening between CS and US in autoshaping. Joumal of Experimental Psychology: Animal Behavior Processes, 8, 131-141.

Royalty, P., Williams, B. A., Fantino, E. (1987). Effects of delayed conditioned reinforcement in chain schedules. Journal of the Experimental Analysis of Behavior, 47, 41-56.

SChaAl, D. W., \& Branch, M. N. (1988). Responding of pigeons under variable-interval schedules of unsignaled, briefly signaled, and completely signaled delays to reinforcement. Joumal of the Experimental Analysis of Behavior, 50, 33-54.

SChaAl, D. W., Branch, M. N. (1990). Responding of pigeons under variable-interval schedules of signaled-delayed reinforcement: Effects of delay-signal duration. Journal of the Experimental Analysis of Behavior, 53, 103-121.

SPENCE, K. W. (1947). The role of secondary reinforcement in delayed reward learning. Psychological Review, 54, 1-8.

Thomas, G. V., Lieberman, D. A. (1980). Commentary: Determinants of success and failure in experiments on marking. Learning \& Motivation, 21, 110-124.

Thomas, G. V., Lieberman, D. A., McIntosh, D. C., Robertson, P. (1983). The role of marking when reward is delayed. Journal of Experimental Psychology: Animal Behavior Processes, 9, 401-411.

Thomas, G. V., Robertson, D. , Cunniffe, G. (1989). Facilitation of responding in a filled-delay trace autoshaping procedure: An occasion-setting hypothesis. Learning \& Motivation, 20, 225-241.

Thomas, G. V., Robertson, D., Lieberman, D. A. (1987). Marking effects in Pavlovian trace conditioning. Joumal of Experimental Psychology: Animal Behavior Processes, 9, 401-411.

UrcuioL, P. J., \& KASPROW, W. J. (1988). Long-delay learning in the T-maze: Effects of marking and delay-interval location. Learning \& Motivation, 19, 66-86.

WiLlums, B. A. (1971). The effects of intertrial interval on discrimination reversal learning in the pigeon. Psychonomic Science, 23, 241-243.

Williams, B. A. (1981). Invariance in reinforcements to acquisition, with implications for the theory of inhibition. Behaviour Analysis Letters, 1, 73-80.

Williams, B. A., Dunn, R. (1991a). Preference for conditioned reinforcement. Journal of the Experimental Analysis of Behavior, 55, 37-46.

Williams, B. A., \& DuNN, R. (1991b). Substitutability between conditioned and primary reinforcers in discrimination acquisition. Journal of the Experimental Analysis of Behavior, 55, 21-35.

(Manuscript received November 19, 1990; revision accepted for publication May 21, 1991.) 